Somnologie 2010 • 14:85-86

DOI 10.1007/s11818-010-0473-x

Online publiziert: 4. Juni 2010

(c) Springer-Verlag 2010

\author{
G. Mayer \\ Hephata-Klinik, Schwalmstadt-Treysa
}

\title{
Schichtarbeit und Schlafstörungen
}

immer wieder mit der tristen Realität der Schichtarbeit.

Dennoch können und dürfen wir uns dieser Realität der 24-h-Gesellschaft nicht verschließen, müssen sie bis zu einem gewissen Punkt akzeptieren und können oft nur versuchen, den Betroffenen den Umgang mit der Schichtarbeit soweit wie möglich zu erleichtern. Erstaunlicherweise hat das letzte Jahrhundert noch Rücksicht auf die Bedürfnisse von Schichtarbeitern genommen. Damals wurden in Regionen mit Schichtarbeit Häuser konzipiert, in denen die Arbeitenden für den Tagesschlaf Schlafräume zur Verfügung hatten, die - ohne den Rest der Familie zu beeinträchtigen - so gelegen waren, dass sie ein Minimum an Geräusch- oder Lichtbelästigung boten. Die Betriebsmediziner sind sich über das Spektrum der die Schichtarbeit flankierenden Probleme im Klaren und haben diese auch weitestgehend ausgeleuchtet. Allerdings ist die Hilflosigkeit, die sich in den mangelnden Umsetzungsmöglichkeiten spiegelt, erschreckend. Für die Hausärzte und die Schlafmediziner, die sich mit den Schlafproblemen der Schichtarbeiter(innen) beschäftigen müssen, stehen auch nicht viele Möglichkeiten zur Verfügung.

\section{( Wir können und dürfen uns dieser Realität der 24-h- Gesellschaft nicht verschließen}

Wir wissen genau, dass viele unserer Patienten, wenn wir ihnen nicht helfen können, ihre Schichtarbeit weiter zu verrichten, arbeitslos werden würden. Das können und wollen wir nicht riskieren. Also haben wir ein "Management" entwickelt, das daher schon vom Ansatz her recht kläglich sein muss. Die theore- tischen Grundlagen dieses Managements sind - wie uns Hajak u. Rodenbeck zeigen - plausibel und zumindest teilweise evidenzbasiert, allerdings meist nicht auf langfristiger Beobachtung basierend oder gar die Lebensqualität der Betroffenen berücksichtigend. Es existieren immer noch keine prospektiven Untersuchungen, die uns zeigen, wie sich ein Schichtarbeiterleben oder dessen Gesundheit im Verlauf von beispielsweise 10 Jahren verändert. Die wenigen Modelle, die mit sinnvollen Schichten umgesetzt wurden, haben sich nicht etabliert.

Gleichzeitig treibt der verbreitete Personalmangel bei parallelem PersonalEinsparungsdruck den „Wahnsinn“ des Schichtdienstes bei Polizei, Pflegepersonal, Bus- und Bahnverkehr auf die Spitze. Wir sehen diese Betroffenen täglich in unserer Praxis und sind dazu verdammt, sie entweder „kaputtzuschreiben“ oder medikamentös zumindest eine Zeit lang über Wasser zu halten. Sogar die Erwartung dieser Menschen, mit Rentenbeginn endlich wieder ordentlich schlafen zu können, müssen wir eigentlich auch enttäuschen. Trotzdem ist es gut, dass es einige Medikamente gibt, die es ermöglichen, das Elend eine Zeit lang zu verbessern. Aber es muss uns immer bewusst bleiben, dass wir das Schichtarbeitersyndrom - genauso wie viele andere Erkrankungen - nicht ursächlich heilen, sondern nur lindern können. Und wir müssen uns gutachterlich mit den Folgen der Schichtarbeit auseinandersetzen, meist retrospektiv, nicht prospektiv. Diesem Problem ist daher ein eigener Beitrag gewidmet.

Trotz, oder gerade wegen, der genannten Einschränkungen und Probleme bei der Umsetzung theoretisch fundierter Grundlagen in den klinischen Alltag und 
der viel zu großen Zahl der von Schichtarbeit betroffenen Personen ist dieses Editorial nicht als Grabstein für die Schlafmedizin in der Schichtarbeit zu verstehen. Im Gegenteil! Das Editorial wie das ganze Themenheft soll die Schlafforschung und Schlafmedizin dazu animieren, sich inhaltlich intensiver und langfristiger diesem Problem zu widmen, um es den Betroffenen und den Gesundheitsinstitutionen immer wieder vor Augen zu führen. Dabei dürfen wir uns nicht nur in den interessanten Aspekten der Messung von Müdigkeit, Schläfrigkeit und Fatigue verlieren, sondern müssen vielmehr Konzepte entwickeln, die sozial verträgliche Arbeitszeiten, Pausen, Ernährungskonzepte an der Arbeit und damit ein geringeres Morbiditäts- und Mortalitätsrisiko für die Betroffenen und vermindertes Unfallrisiko für die Gesellschaft ermöglichen. In diesem Sinne hoffe ich auf die Fortschritte in den nächsten 20 Jahren, die translational auch gesellschaftlicher Natur sein sollten.

\section{Prof. Dr. Geert Mayer}

\section{Korrespondenzadresse}

Prof. Dr. G. Mayer

Hephata-Klinik

Schimmelpfengstraße,

34613 Schwalmstadt-Treysa

dgsm.mayer@t-online.de

\section{Der Dachverband für alle Schlafgestörten - BSD}

Der Bundesverband Schlafapnoe und Schlafstörungen Deutschland e.V. (BSD) hat sich neu orientiert: Er hat einen neuen Vorstand gewählt und die Weichen für die Zukunft gestellt. Von nun an setzt sich der BSD nicht nur für Betroffene mit Schlafapnoe ein,sondern bietet auch all denen Unterstützung an, die unter verschiedensten Arten von Schlafstörungen leiden. Der Verein wirkt gleichzeitig als Dachverband für die bundesweiten Selbsthilfegruppen.

Die überregionale Arbeit geht weit über den bloßen Informationsaustausch hinaus: Es werden Schulungen, Kongresse und Vorträge organisiert, und die Selbsthilfegruppen mit Print- und audiovisuellen Medien bei ihrer Arbeit vor Ort unterstützt. „Die Öffentlichkeit weiß noch immer sehr wenig über Schlafprobleme und tut sie leider immer noch oft als banal $a b^{\prime \prime}$, erklärt der neue Vorsitzende des Bundesverbandes, Werner Waldmann. „Das muss sich ändern. Es muss noch viel mehr für die Betroffen getan werden, und wir müssen die Bedeutung von Schlafapnoe und Schlafstörungen weiter ins Bewusstsein der Öffentlichkeit tragen. Gestörter Schlaf ist nicht lediglich eine Befindlichkeitsstörung, sondern kann Lebensqualität und Gesundheit der Betroffenen erheblich beeinträchtigen. Außerdem gehen viele Unfälle in Beruf und Straßenverkehr auf sein Konto."

Werner Waldmann hat das Patientenmagazin "das schlafmagazin" begründet. Stellvertretender BSD-Chef ist der Schlafmediziner Professor Karl-Heinz Rühle aus Hagen, der als Mitglied der Deutschen Gesellschaft für Schlafforschung und Schlafmedizin (DGSM) für die fachliche Kompetenz der Aktivitäten des Bundesverbandes garantiert. Als zweiter Stellvertreter wurde Professor Matthias Leschke vom Klinikum Esslingen a.N. gewählt: Er bringt als Kardiologe seine Kompetenz in Sachen Herz-Kreislauf-Erkrankungen in den Bundesverband ein. Ein Beirat aus hochkarä- tigen Medizinern und Juristen unterstützt die Arbeit des Vorstands. Der Schlafexperte Professor Jürgen Zulley wird den Bundesverband als Botschafter unterstützen.

Pressestelle BSD

Panoramastraße 6

D-73760 Ostfildern 